\title{
Hommage à Marc Boyer (1926-2018)
}

\section{Philippe Clairay et Johan Vincent}

\section{(2) OpenEdition}

\section{Journals}

Édition électronique

URL : http://journals.openedition.org/tourisme/2265

DOI : $10.4000 /$ tourisme 2265

ISSN : 2492-7503

\section{Éditeur}

Éditions touristiques européennes

Référence électronique

Philippe Clairay et Johan Vincent, « Hommage à Marc Boyer (1926-2018) », Mondes du Tourisme [En ligne], 15 | 2019, mis en ligne le 01 juin 2019, consulté le 15 septembre 2020. URL : http:// journals.openedition.org/tourisme/2265

Ce document a été généré automatiquement le 15 septembre 2020

\section{cc) (i) (9)}

Mondes du tourisme est mis à disposition selon les termes de la licence Creative Commons Attribution - Pas d'Utilisation Commerciale - Pas de Modification 4.0 International. 


\title{
Hommage à Marc Boyer (1926-2018)
}

\author{
Philippe Clairay et Johan Vincent
}

1 Le 6 août 2018 disparaissait l'historien Marc Boyer, à l'âge de 92 ans. Spécialiste des villégiatures (thermales, balnéaires et hivernales), cet homme, très érudit, a été pionnier dans l'étude du phénomène touristique et de ses répercussions sur la société, avec l'essor des loisirs et la création de nouveaux modes de vie. Toujours à l'affût des nouveaux usages et des nouvelles pratiques, il laisse une œuvre écrite considérable, dont le point d'orgue est sa thèse d'État consacrée à « l'invention du tourisme dans le Sud-Est de la France ».

2 Une riche carrière. Après de brillantes études d'histoire, couronnées par l'obtention de l'agrégation, Marc Boyer fait du tourisme, et de l'histoire de son invention, le grand sujet de sa vie. Il s'implique très rapidement dans des projets concrets. Dès 1957, il suggère diverses améliorations au directeur général du Tourisme et aux responsables de l'Insee (Boyer, 1962). L'enquête par sondage sur les départs de l'Insee de 1961 est ainsi étendue à l'ensemble des Français (et non plus seulement à dix-huit grandes villes françaises) et appréhende mieux les raisons du départ (plage, neige...) et les régions de départ.

3 En 1960, Marc Boyer est attaché de recherches au CNRS à Marseille. Il fonde en 1961 le Centre d'études du tourisme à l'Université d'Aix-Marseille. Devenu secrétaire général de cet organisme, il organise plusieurs colloques scientifiques, dont un en 1962, à Nice, sur les mutations des saisons touristiques. Le Centre publie alors les Cahiers du tourisme, au moins jusqu'en 1974. En 1975, il devient le Centre des hautes études touristiques de la faculté d'Aix-en-Provence. Maître de conférences à l'Université Lumière-Lyon 2 en 1991, puis vice-président de cette université, il y fonde le département de tourisme, première UFR consacrée à ce thème en France. Il est aussi membre du Conseil national du tourisme.

4 Son expertise était aussi reconnue à l'échelle internationale, comme en témoignent ses nombreuses missions à l'étranger. Il devient ainsi président de l'Association méditerranéenne de sociologie du tourisme (fondée en 1988) à la fin des années 1990. En décembre 2011, il est appelé à prendre la présidence du Comité national d'histoire $\mathrm{du}$ tourisme au sein de l'Institut français du tourisme. L'ambition est de lancer divers 
guides, dont un consacré aux sources sur l'histoire du tourisme, de promouvoir un appel à propositions de recherche, de lancer le Prix Jacques-Marseille et de préparer des collections d'ouvrages et des projets d'exposition. Cela sera fait en partie, avec le lancement de la collection d'ouvrages "Tourisme, compétences et métiers" chez De Boeck et l'organisation d'un prix sous la forme d'un concours de prospective touristique ouvert aux étudiants en tourisme.

Ses dernières contributions ont été pour le dossier de classement Unesco de la ville de Nice. L'exposition "Nice ou l'invention du tourisme» a été présentée au cours de l'année 2018 en France et à l'étranger (à l'Institut français de Bulgarie en mars 2018, par exemple).

6 Une production scientifique majeure. L'une des caractéristiques des travaux de Marc Boyer est d'opérer un va-et-vient permanent entre l'histoire et l'actualité. En effet, pour lui, l'histoire est en permanence présente pour expliquer les paysages, les équipements, les habitudes des touristes, les renommées, et ce jusqu'aujourd'hui. Pour cette raison, dans ses différents ouvrages, comme Le Tourisme (1972) ou L'Invention du tourisme (1996), il s'appuie sur la description de la résidence oisive des $\mathrm{XVIII}^{\mathrm{e}}$ et $\mathrm{XIX}^{\mathrm{e}}$ siècles afin de comprendre les mutations sociales qui se sont opérées au cours des décennies suivantes - jusqu'à la frustration de ceux qui ne partent pas en vacances. Il a notamment contribué à saisir l'originalité des pratiques sociales qui s'opèrent au cours de ces deux siècles, les distinguant des pratiques touristiques de Montaigne ou des acteurs du Grand Tour (dans lequel le tourisme d'agrément puiserait tout de même certaines de ses racines). Activité multiforme, le tourisme ne pouvait qu'épanouir l'esprit curieux de Marc Boyer, qui ne se cantonnait pas aux seuls enjeux historiques.

7 C'est notamment en abordant l'aspect protéiforme du tourisme qu'il en a profondément renouvelé l'approche. Chacune des différentes approches sectorielles se prétendait au cœur du tourisme: les sociologues sur les effets déstructurants, les économistes sur l'effet multiplicateur dans les économies locales, les géographes sur la «touristification » des espaces, le management sur les hôtels, les historiens d'art sur les musées, les Staps sur le tourisme sportif... C'est cet ensemble qu'il fallait conjuguer, en prenant garde de ne pas tomber dans le contre-sens contextuel. Les diverses formes du tourisme ont été inventées et ses pratiques peuvent souffrir d'obsolescence. Ses attraits ne sont pas des données mais des agents socio-culturels. Le schéma marketing d'une offre répondant à une demande est réducteur.

Marc Boyer a notamment défendu une étude du tourisme dénuée d'a priori négatif. Il regrettait que cette étude soit traitée «comme un wagon de queue ». À l'aune d'un territoire, le tourisme vient perturber et détruire le quotidien des populations dites traditionnelles : il ne peut donc qu'être finalement mauvais (Boyer, 2002). Il prônait le pas de côté, pour au contraire tenter de décrire le "sens » du tourisme (et non le sens de l'Histoire). Il avait souligné et démontré le rôle des gate-keepers, esprits originaux qui ont proposé des pratiques alternatives et transmis de nouveaux modes de vie. Les études comportementales sont à la base de ses études. Il a ainsi montré que le tourisme, loin de détruire le monde, le recompose, le renouvelle, le revivifie. Ainsi, le tourisme du début du Xxe siècle n'entre pas dans les catégories des années 1960 (culture et loisirs de masse), mais suit le même processus dialectique: invention de distinction par les marginaux, consécration par les stars, diffusion par imitation capillaire, nouvelles inventions pour remplacer des pratiques et des lieux devenus trop communs (Boyer, 1997). 
9 Auteur de nombreux articles (son premier article date de 1958, pour les actes du $83^{\mathrm{e}}$ congrès des sociétés savantes, parus dans Géographie) et ouvrages au cours de sa carrière, ses publications deviennent plus nombreuses à partir du milieu des années 1990. Cette période correspond à la soutenance de sa thèse d'État, en 1997, sur l'invention du tourisme dans le Sud-Est; thèse publiée en 2000. S'il a conservé un fort attachement à cette région, qu'il a longuement étudiée dans le cadre universitaire, il a ouvert largement ses perspectives géographiques, jusqu'au monde entier. En 2006, il est distingué par l'Académie française, qui lui décerne son Prix Guizot pour Le Thermalisme dans le Grand Sud-Est de la France, paru aux Presses universitaires de Grenoble.

Marc Boyer a beaucoup marqué les étudiants qu'il a pu croiser. Chevalier de la Légion d'honneur et chevalier des Arts et des Lettres, il a également reçu les hommages de la Nation.

\section{BIBLIOGRAPHIE}

\section{Bibliographie sélective :}

Marc BOYER, Le Tourisme, Seuil, «Peuples et Cultures », 1972, 262 p.

Marc BOYER, L'invention du tourisme, Gallimard, « Découvertes Gallimard », 1996, 160 p.

Marc BOYER, L'invention du tourisme. Origine et développement du tourisme dans le Sud-Est de la France, du XVIe à la fin du Second Empire, thèse de doctorat d'État en lettres et sciences humaines, Université de Lyon II, 1997, 5 vol. , 2360 p., publiée aux Éditions de l'Aube en 2000 sous le titre Histoire de l'invention du tourisme dans le Sud-Est de la France, XVIe-XIXe.

Marc BOYER, Histoire du tourisme de masse, PUF, « Que sais-je ? », 1999, 128 p.

Marc BOYER, L'invention de la Côte d'Azur : l'hiver dans le Midi, Éditions de l'Aube, préface de Maurice Agulhon, 2002, 378 p.

Marc BOYER, Histoire générale du tourisme du XVIe siècle au XXIe siècle, L'Harmattan, 2005, 327 p.

Marc BOYER, Le Thermalisme dans le Grand Sud-Est de la France, Presses Universitaires de Grenoble, $2005,420 \mathrm{p}$.

Marc BOYER, Ailleurs : histoire et sociologie du tourisme, L'Harmattan, 2011, 301 p.

\section{Références :}

Marc BOYER, «La géographie des vacances des Français », Revue de Géographie alpine, 1962.

Marc BOYER, « Le tourisme, une épistémologie spécifique en quête de paradigmes », Society and Leisure, vol. 20, 1997.

Marc BOYER, « Comment étudier le tourisme ?», Ethnologie française, n 3, vol. 32, 2002. 
AUTEURS

PHILIPPE CLAIRAY

Docteur en histoire

Directeur des Musées et du Patrimoine

Ville de Villedieu-les-Poêles-Rouffigny

\section{JOHAN VINCENT}

Docteur en histoire, post-doc Esthua (Université d'Angers)

Chercheur associé au TEMOS (CNRS FRE 2015), Université de Bretagne-Sud 